\title{
A new double imaging velocity focusing coincidence experiment: $i^{2}$ PEPICO
}

\author{
Andras Bodi, ${ }^{1, \text { a) }}$ Patrick Hemberger, ${ }^{1}$ Thomas Gerber, ${ }^{1}$ and Bálint Sztáray ${ }^{2}$ \\ ${ }^{1}$ Paul Scherrer Institut, Villigen 5232, Switzerland \\ ${ }^{2}$ Department of Chemistry, University of the Pacific, Stockton, California 95211, USA
}

(Received 10 July 2012; accepted 21 July 2012; published online 13 August 2012)

\begin{abstract}
The vacuum ultraviolet (VUV) beamline of the Swiss Light Source has been upgraded after two years of operation. A new, turntable-type monochromator was constructed at the Paul Scherrer Institut, which allows for fast yaw-alignment as well as quick grating change and exchange. In addition to the original imaging photoelectron photoion coincidence endstation ( $i$ PEPICO), a second, complementary double imaging setup $\left(i^{2}\right.$ PEPICO) has been built. Volatile samples can be introduced at room temperature or in a molecular beam, a pyrolysis source allows for radical production, and non-volatile solids can be evaporated in a heated cell. Monochromatic VUV radiation ionizes the sample and both photoelectrons and photoions are velocity map imaged onto two fast position sensitive detectors and detected in delayed coincidence. High intensity synchrotron radiation leads to ionization rates above $10^{5} \mathrm{~s}^{-1}$. New data acquisition and processing approaches are discussed for recording coincidence processes at high rates. The setup is capable of resolving pulsed molecular beam profiles and the synchrotron time structure temporally. The latter is shown by photoelectron autocorrelation, which displays both the $1.04 \mathrm{MHz}$ ring clock frequency as well as resolving the micro-pulses with a separation of $2 \mathrm{~ns}$. Kinetic energy release analysis on the dissociative photoionization of $\mathrm{CF}_{4}$ indicates a dissociation mechanism change in the Franck-Condon allowed energy range of the first ion state. (C) 2012 American Institute of Physics. [http://dx.doi.org/10.1063/1.4742769]
\end{abstract}

\section{INTRODUCTION}

Imaging in general, velocity map imaging (VMI ${ }^{1}$ and its variation, direct current slice imaging ${ }^{2}$ in particular, belong among the most versatile tools in chemical physics. While ion imaging was first proposed to study chemical reactions, ${ }^{3}$ electron velocity map imaging has gained widespread use, as well. ${ }^{4,5}$ Two- and multiparticle imaging techniques such as cold target recoil ion momentum spectroscopy ${ }^{6}$ and velocity imaging photoionization coincidence ${ }^{7}$ have emerged to obtain velocity vector correlations in, e.g., dissociative photoionization. ${ }^{8,9}$ Most of these experiments are pulsed, which restricts energy selection in non-resonant excitation, and very few work in the valence photoionization regime. At the same time, similar to the setup described herein, some of them use double velocity map imaging with time of flight analysis to study, e.g., angular correlations. In recent years, several newly constructed single imaging coincidence endstations were also commissioned at synchrotron beamlines, ${ }^{10-12}$ among them, the $i$ PEPICO endstation ${ }^{13}$ at the vacuum ultraviolet (VUV) beamline ${ }^{14}$ of the Swiss Light Source. Here, we present the $i^{2}$ PEPICO apparatus, a double imaging setup complementary to the $i$ PEPICO equipment, which offers electron and photoion imaging with improved optical characteristics to study dissociative photoionization.

In laser-based experiments, ultrafast phenomena can be studied by photoelectron imaging, ${ }^{15}$ and state selection ${ }^{16}$ can be achieved by resonant photoexcitation. Photoelectron angular distribution ${ }^{17}$ and VUV circular dichroism experiments $^{18,19}$ are among the main incentives to develop

a) Author to whom correspondence should be addressed. Electronic mail: andras.boedi@psi.ch. photoelectron imaging apparatuses at synchrotron VUV light sources. Although state selection is sometimes referred to in continuous photoionization coincidence experiments, ${ }^{20,21}$ the photoionization and, particularly, threshold photoionization mechanisms are complicated and often comprise competing parallel pathways. ${ }^{22-24}$ It is difficult to establish exact state specificity in such experiments. However, one of the enticing aspects of describing state-specific dissociation pathways is the possibility to unveil couplings between different degrees of freedom and to understand the role of potential energy surfaces in channeling the reactive flux in the fast initial stages of the dissociation. ${ }^{25}$

One-photon threshold ionization using tunable radiation has two distinct benefits. First, excitations to the quasicontinuous Rydberg continuum of a multi-atom molecule are almost always allowed, leading to the filling-up of the threshold photoelectron spectrum in the Franck-Condon gaps. ${ }^{26}$ All energy levels are, thus, accessible, including Franck-Condon gaps inaccessible by direct (non-threshold) photoionization. The only exceptions encountered so far involve very broad Franck-Condon gaps, which presumably lead to a depletion of the Rydberg continuum. ${ }^{27}$ Second, threshold electrons can easily be selected for as proposed by Sztáray and Baer, ${ }^{28,29}$ which allows for internal energy selection in the corresponding photoions. Thus, by scanning the photon energy, and, consequently, the parent ion internal energy, the photoion dissociation pathways can be studied by mass analysis. ${ }^{30}$ Using low ion extraction fields and large ion residence times in the $i$ PEPICO apparatus yields unimolecular dissociation rates in the $10^{3}-10^{7} \mathrm{~s}^{-1}$ range for metastable parent ions, which can be modeled ${ }^{31}$ to extract dissociative photoionization energies and thereby other thermochemical data with sub-kJ mol ${ }^{-1} \mathrm{ac}-$ curacy in favorable cases. ${ }^{23}$ 
Continuous ion imaging experiments in the VUV range are still scarce in comparison with electron imaging ones. In the dissociative photoionization of ethane, Li et al. imaged the ion signal and suppressed the detector area where parent ion contributions were assumed. ${ }^{32}$ In a more recent study ${ }^{33}$ Gans et al. determined the absolute photoionization cross section of the ethyl radical and used ion imaging in the DELICIOUS II experiment ${ }^{10}$ as a tool to tell ethyl ions as dissociative photoionization products apart from photoionized ethyl radicals. Mahjoub et al. have also used ion imaging to show the absence of kinetic energy release (KER) in a slow photoelectron study of $\delta$-valerolactam and ascertain that the dissociative photoionization of heavier adducts did not perturb the photoelectron spectrum of the lighter ones. ${ }^{34}$ These studies rely on kinetic energy release in unimolecular dissociation reactions to identify dissociation product signal or a lack thereof.

The experiment described here is unique in the sense that it allows for valence photoionization experiments at high ionization rates with simultaneous electron and ion velocity map imaging in delayed coincidence. Electrons provide the start signal for the ion time-of-flight in continuous experiments, and their detection is required in TOF mass analysis. As will be shown later, by applying the same subtraction scheme to select threshold electrons as in the $i$ PEPICO experiment, ${ }^{28}$ it is easily possible to obtain ion images for internal energy selected photoions. This opens the way to (1) quantitative and high resolution kinetic energy release analyses as a function of parent ion internal energy, and (2) background free molecular beam measurements, as the thermal component of the ion signal is spatially separated from the cold one,${ }^{34}$ improving the accuracy of onset energy determinations. ${ }^{35}$ As ion internal energy selection and continuous scanning becomes possible in ion imaging experiments, we also expect to observe state-specific phenomena. Photoion kinetic energy release and angular distribution measurements will make it possible to identify concurrent decay processes routinely, e.g., in and in between threshold photoelectron peaks. We entertain the thought of relating anisotropies in photoion angular distributions to the competition between dissociation and molecular rotation.

A third prospective use is related to the symmetric construction of the $i^{2}$ PEPICO experiment. By applying two grids on each side, first order space focusing conditions ${ }^{36}$ can be achieved for both positively and negatively charged species. The ratio of the coincident $\mathrm{Ar}^{+}$photoion signal over the total photoelectron signal slightly above the ionization energy yields the absolute ion collection efficiency, which is typically in the 15\%-20\% range. Assuming an ion collection efficiency of $20 \%$, this would allow for a $4 \%$ overall collection efficiency for anion-cation pair formation in positive ion-negative ion coincidence (PINICO) experiments. ${ }^{37,38}$ Ion pair states have been studied in small molecules, ${ }^{39-41}$ the most fruitful approach at synchrotrons being anion spectroscopy so far. ${ }^{42-44}$ The difficulty of ion pair experiments arises from the generally poor nuclear wave function overlap between the molecular ground state and the lowest lying ion pair states. Because of the higher flux and number densities in the ionization region, PINICO experiments could be possible for a wider range of small molecules than previously investigated. Such studies will be extended to include large species with ion pair ground states, too.

In Sec. II, we first describe a new, plane grating grazing incidence monochromator that enables easy yaw alignment and swift grating exchange and provides a flexible flux vs. resolution compromise according to experimental needs. We will then introduce the double imaging setup and discuss data acquisition and processing aspects, which will be illustrated by molecular beam pulse shape measurements and photoelectron autocorrelation traces. We conclude by presenting new kinetic energy release data for the $\mathrm{CF}_{4}+h v$ $\rightarrow \mathrm{CF}_{3}{ }^{+}+\mathrm{F}+\mathrm{e}^{-}$process.

\section{EXPERIMENTAL}

\section{A. Monochromator}

The optical setup of the VUV beamline has been detailed in a previous publication with special emphasis on the compact rare gas filter to suppress higher harmonic radiation. ${ }^{14}$ The grating yaw alignment, previously not possible in-vacuum, tedious grating change, and almost impossible grating exchange have proved to be limiting factors in the performance of the beamline. Therefore, we decided to build an in-house designed, turntable-type plane grating monochromator with an increased scanning range while keeping the collimating and focusing mirrors as well as the original laminar gratings from Horiba Jobin Yvon.

The design goals of the new improved monochromator were (1) greater adjustment ranges for in-vacuum alignment to relax the out-of-vacuum pre-alignment requirements, (2) increased pitch angle scanning range from $\pm 10^{\circ}$ to $\pm 15^{\circ}$ to increase the energy range, (3) reproducible switch between gratings under ultrahigh vacuum conditions, and (4) easy grating exchange.

At first, only the grating cradles were redesigned, which could have been installed in the existing monochromator, ${ }^{14}$ meeting only the first criterion, albeit the most important one.

In order to meet all four goals, we decided to build a completely new monochromator chamber comprising the grating cradles mounted on a precision rotation stage from Micos (PRS-200), driven by an in-vacuum stepper motor, as shown in Figure 1. The pitch axis, i.e., the energy scan direction is defined by ceramic ball bearings. The scanning movement is driven by an out-of-vacuum linear stage acting on a vacuum feedthrough. The connection between the linear stage and the rotating sinus arm consists of flexural pivot bearings. Similar to the old chamber, the pitch angle is read out directly using a Renishaw optical encoder with a glass ruler mounted on a circle around the pitch axis. The new grating cradles, shown in Figure 2, allow roll adjustment in the $\pm 1.5 \mathrm{mrad}$ range with $\pm 6 \mu$ rad precision as well as yaw adjustment in the $\pm 2.5 \mathrm{mrad}$ range with $\pm 3 \mu \mathrm{rad}$ precision.

The new chamber and grating cradles together with new, in-house designed and manufactured vacuum chamber platforms were commissioned within three days comparing favorably with the 3 months needed for the original setup. The new monochromator also fulfills the fourth design aim, proven by the installation of a new ruled 150 groove/mm gold-coated 

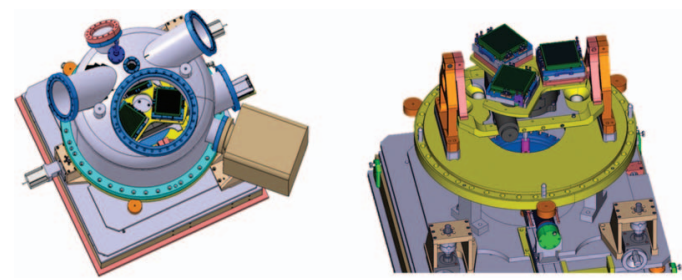

FIG. 1. Turntable-type monochromator showing the precision rotation stage below the grating change dial from top (left) and shown without the vacuum chamber (right).

grating blazed at an angle of $1.29^{\circ}$ from Richardson gratings with the monochromator only vented for a few hours. Preliminary measurements indicate that this grating delivers 6-8 times more flux at a photon energy of $8-10 \mathrm{eV}$ at a resolving power of $\sim 1000$. The increased flux is most welcome in studies of transient species, e.g., radicals produced in flash pyrolysis. ${ }^{45-47}$

\section{B. Symmetric double velocity map imager}

The double imaging setup was realized as an extension to the $i$ PEPICO apparatus, ${ }^{13}$ and the aim was to reuse as many components as possible (Figure 3 ). The source chamber with the $50001 \mathrm{~s}^{-1}$ cryogenic Leybold COOLVAC $5000 \mathrm{CL}$ and $1600 \mathrm{l} \mathrm{s}^{-1}$ turbomolecular Pfeiffer TPH 1201 UP pumps was kept unchanged. The $i$ PEPICO experimental chamber is pumped by a $1500 \mathrm{l} \mathrm{s}^{-1}$ COOLVAC $1500 \mathrm{CL}$ cryopump and a $5001 \mathrm{~s}^{-1}$ Pfeiffer TMH 521 YP turbomolecular pump. The smaller size of the $i^{2}$ PEPICO ionization chamber allows mounting only one of the two pumps. The high vacuum pumps are backed by Adixen ACP 28G and ACP 40G Roots pumps. The endstation is O-ring sealed throughout with the exception of the detector flanges. The end vacuum in the interaction and source chambers is in the low $10^{-7}$ mbar range. Typical working pressures are 2-6 $\times 10^{-6}$ mbar in the ionization chamber and $10^{-5}-10^{-4}$ mbar in the source chamber during molecular beam experiments.

The bending magnet synchrotron radiation with virtual light source dimensions of $45 \mu \mathrm{m}(\mathrm{v})$ and $185 \mu \mathrm{m}(\mathrm{h})$ defines the entrance slit of the beamline. ${ }^{14}$ The synchrotron radiation is collimated by a mirror, dispersed vertically by a plane grating in the monochromator (vide supra) and guided through the gas filter by a focusing mirror. The 100-1000 $\mu \mathrm{m}$ (v) exit
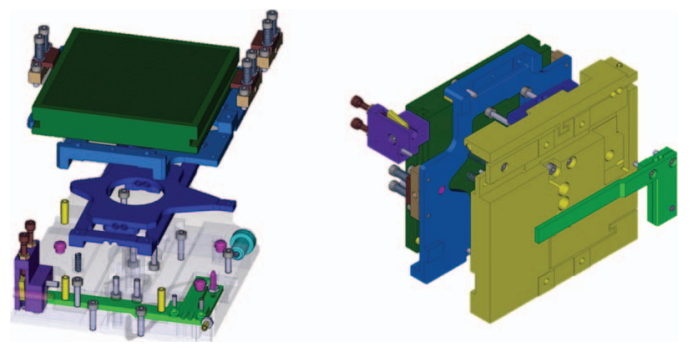

FIG. 2. New grating cradle for the Jobin Yvon $1200 \mathrm{~mm}^{-1}$ and $600 \mathrm{~mm}^{-1}$ as well as the Richardson $150 \mathrm{~mm}^{-1}$ plane gratings. The precision yaw and roll adjustment screws are seen in the lower left corner (left, side view) and upper left corners (right, back view).
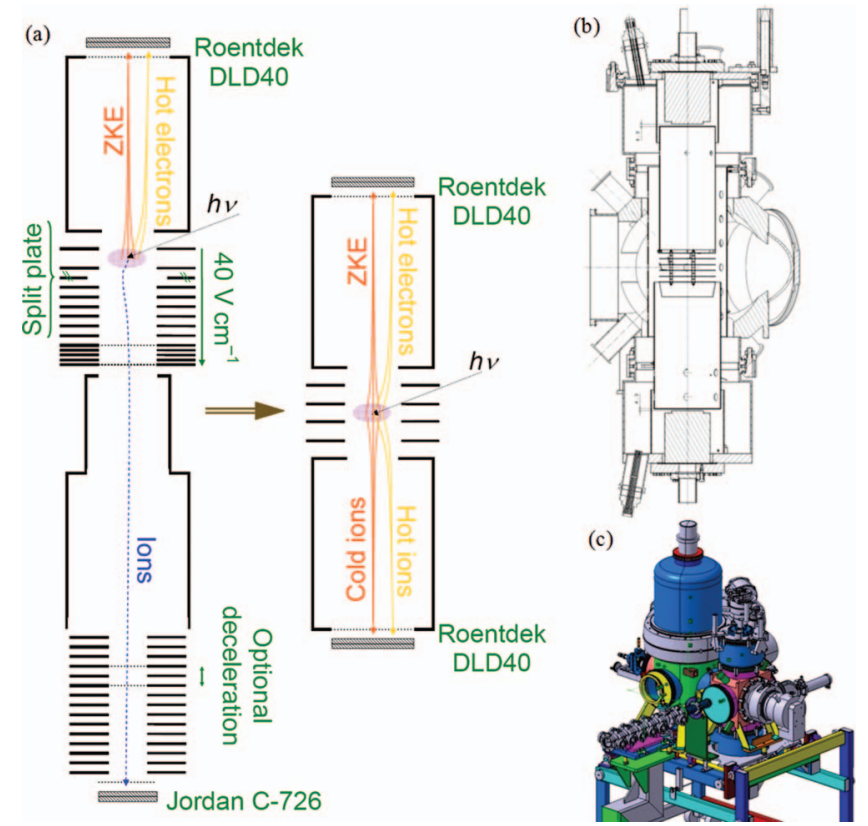

FIG. 3. (a) Schematic representation of the changes from the velocity map imager/TOF analyzer $i$ PEPICO setup to the double velocity map imager $i^{2}$ PEPICO experiment. (b) Drawing of the actual experiment and (c) the setup shown from light beam entrance side together with the gas filter.

slit for the dispersed light is defined by the focus toward the end of the $1 \mathrm{~m}$ long gas filter, $50 \mathrm{~cm}$ upstream from the ionization region. The relatively large, $4 \times 8 \mathrm{mrad}^{2}$ divergence of the light beam thus leads to a $2 \times 4 \mathrm{~mm}^{2}$ maximal spot size in the ionization chamber, which can be decreased by closing the beam apertures in the beamline front end, $3 \mathrm{~m}$ from the bending magnet light source. The large spot size has not affected the velocity map imaging of threshold electrons onto a center spot in the detector, a prerequisite for threshold PEPICO experiments, and only leads to an acceptable broadening of the ion TOF peaks. In SIMION simulations, we found that velocity map focusing conditions for kinetic electrons and ions can be much improved by adding an extra electrode for correcting aberrations owing to the large spot size. Therefore, we added one more plate in the extraction region on both sides. The distance between the plates measures $12 \mathrm{~mm}$. The electrodes have openings of 8,20 , and $15 \mathrm{~mm}$, in the flight direction. The flight tube diameter has been increased from 50 to $116 \mathrm{~mm}$ to improve imaging. Ion optics simulations indicate a resolution of $<2.5 \% \Delta E / E$ across most of the detector area, which corresponds to 5-40 meV at the highest imaged energies depending on the extraction field; cf. the $2-15 \mathrm{meV}$ photon energy resolution depending on photon energy and the choice of grating. The image circle could be increased allowing an upgrade with a larger area imaging detector in the future.

Imaging can be realized with an extraction field ranging between $20 \mathrm{~V} \mathrm{~cm}^{-1}$ and $160 \mathrm{~V} \mathrm{~cm}^{-1}$, with the latter corresponding to velocity map imaging $0-1.6 \mathrm{eV}$ kinetic energy species on a $40 \mathrm{~mm}$ diameter detector. The flight axis in the $i$ PEPICO chamber is off-center to minimize the distance between the skimmer and the ionization region in molecular beam experiments. Because of the larger electrode sizes, the ionization region in the new setup had to be shifted 
$50 \mathrm{~mm}$ back to the center of the ionization chamber. In order to shorten the distance between the skimmer and the interaction region, we have replaced the slim valve ${ }^{13}$ separating the source and experimental chambers with a skimmer holder flange and shifted the skimmer $15 \mathrm{~mm}$ closer to the interaction region.

The magnetic shielding for the electrons in the $i^{2} \mathrm{PEPICO}$ apparatus is provided by a cylindrical $\mu$-metal shield in the ionization region. Both flight tubes are made of $\mu$-metal, and there are coaxial overlapping tubular shields around the detectors. Although the detector backs are left unshielded, no aberrations could be observed. Photoelectrons with well-defined kinetic energy, e.g., from autoionizing $\mathrm{Ar}^{*}$ states, can be used to focus and calibrate both images by choosing fields of appropriate polarity. One flight tube is also available in stainless steel construction to allow the destructive deflection of photoelectrons in anion-cation coincidence experiments by magnetic fields.

Two Roentdek DLD40 position sensitive delay line detectors are used to record coincidences (Figure 3). The four delay line signals $\left(x_{1}, x_{2}, y_{1}\right.$, and $\left.y_{2}\right)$ from each detector are processed after constant fraction discrimination by an 8 channel ATR19 unit and recorded by a HPTDC8-PCI card. The position is derived based on the time differences $\Delta x=x_{1}$ $-x_{2}$ and $\Delta y=y_{1}-y_{2}$. The event time stamp corresponds to the average of the four time values of $x_{1}, x_{2}, y_{1}$, and $y_{2}$. The MCP front signal is not used for additional discrimination, because of the limited number of high resolution channels, but we do not expect this to compromise detection as long as the discrimination thresholds are regularly checked and normalized.

As mentioned earlier, the double imaging $i^{2}$ PEPICO setup is complementary to the $i \mathrm{PEPICO}$ experiment. The former can be used to determine reverse barrier heights and excess energy partitioning between the translations and internal degrees of freedom as well as in ion pair experiments, while the latter allows for unimolecular dissociation rate measurements thanks to large ion residence times.

\section{Data acquisition and processing}

The single imaging $i$ PEPICO apparatus ${ }^{13}$ is the first continuous two particle coincidence experiment making use of the triggerless multiple-start/multiple-stop data acquisition technique. $^{29}$ The highest possible event rate is only limited by the bandwidth of the detector system and does not depend on the flight times at all. As threshold electrons only make up a small fraction of the total photoelectron signal high above the ionization energy, a high count rate is required for the production of internal energy selected parent ions with arbitrary energy. The $i$ PEPICO setup, with a locally developed software environment in Embarcadero Delphi and Microsoft Visual $\mathrm{C}++$, has made it possible to record threshold photoionization signal in energy regions, where $>90 \%$ of even the center signal is attributable to hot electron contamination.

In the double imaging experiment, both the start and the stop signals can have spatial coordinates, complicating the efficient storage and retrieval of TOF distributions with TOF and spatial regions of interest (ROIs) in an incrementally developed data acquisition environment. Therefore, new measurement software has been developed, and is now in exclusive use for both single and double imaging experiments. The HPTDC8-PCI card from chronologic $\mathrm{GmbH}$ based on HPTDC chip developed at CERN is operated in the list mode as previously.

One or two sets of four channels each provide the image information. The image coordinates and hit times, as well as an arbitrary set of channel hits with times, are recorded to file or memory. Thus, the experimental data file is a list of detector events, which is processed when loading. A coincidence set can be defined as a sequence of events with time and position ROIs. The first event is either a single channel hit or an image hit with a detector area ROI, each subsequent event has a time window relative to the starting event, and may have a detector area ROI for an image event. For example, the first event is an electron image hit and the second is a single channel ion hit in $i$ PEPICO, whereas both events have spatial coordinates in an $i^{2}$ PEPICO measurement. When processing the data, the program searches for groups of events, which satisfy all the criteria in the coincidence set. If multiple events satisfy the same member of a coincidence criterion set, the preliminary coincidence events are copied or forked, as prescribed by the generalized multiple-stop/multiple-start approach. ${ }^{29}$ If all criteria in the coincidence set have been fulfilled, the coincidence is complete, and arbitrary two constituents can be assigned as start and stop signal in the TOF distribution, with a freely chosen TOF range and a TOF bin size not smaller than $25 \mathrm{ps}$, the time-to-digital converter resolution. Data acquisition and processing can be completely automated using the locally developed Manoscript scripting language. ${ }^{48}$

An external trigger signal can also be recorded. Coincidence sets of three, namely, trigger, photoelectron, and photoion signals, can be used to record time-dependent ion TOF distributions with ns time resolution and unlimited time range with respect to a trigger signal, which could be put to use in chemical dynamics experiments. Measuring the ion kinetic energy by VMI in the $i^{2}$ PEPICO setup adds another observable, a time resolved molecular thermometer. Aside from simple and quick pulse shape measurements as will be shown next, the benefit of such measurements in chemical dynamics studies is that a reaction can be triggered in a pulsed experiment, and the time and temperature profiles can be obtained with ns resolution.

Although inefficient because of the quasi-continuous photon flux, pulsed sample sources can be used to, e.g., increase the maximum backing pressure in molecular beam experiments. In such instances, pulse shape profiles, such as the argon gas pulse profiles obtained in a pulsed pyrolysis source and shown in Figure 4, can be recorded in a matter of minutes by plotting the photoelectron signal (now the 2nd event in the coincidence set) as a function of the elapsed time from the trigger signal (1st event in the coincidence set). By correlating argon ion images instead of just the photoelectron arrival times as 2 nd events, it was also possible to determine which portion of the pulsed molecular beam is efficiently cooled. The pulse shapes and widths vary with different opening times, but because of a combination of how the 


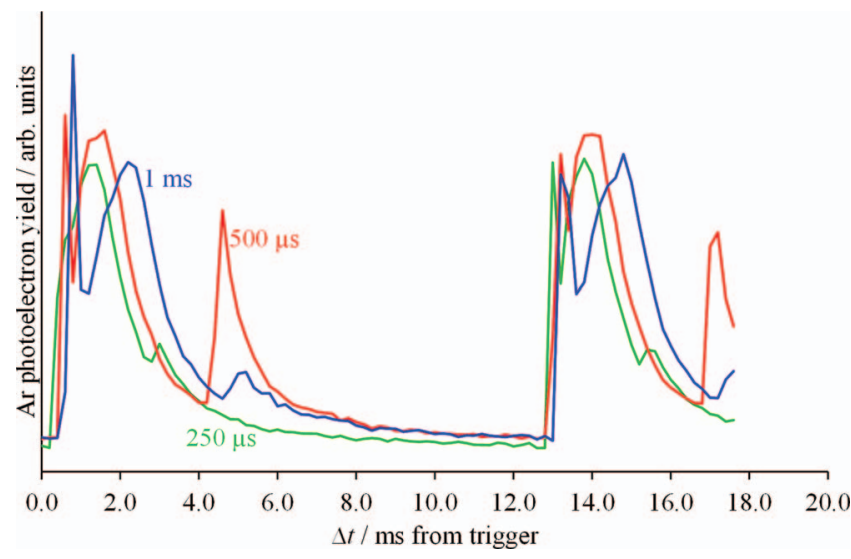

FIG. 4. Argon gas pulse profiles recorded by time-stamping valve open and electron events and plotted with a resolution of $200 \mu$ s. An Iota One ${ }^{\circledR}$ controller was driving a general valve from Parker at $80 \mathrm{~Hz}$ and the opening times were as given in the plot. The source contained a $1 \mathrm{~mm}$ internal diameter $\mathrm{SiC}$ rod for pyrolysis, inducing some pulse broadening. The molecular beam was skimmed by a $1-\mathrm{mm}$ Beam Dynamics skimmer.

poppet bounces back and forth in the valve at high repetition rates and reflections in the silicon carbide tube, the pulse is always quite broad. Only the first, narrow peaks in the $1 \mathrm{~ms}$ and $500 \mu$ s opening time scans have been found to be efficiently cooled.

The flexibility of the approach is further illustrated in Figure 5, which shows the autocorrelation function of photoelectron events. The variations with $0.96 \mu$ s periodicity, plotted in $8 \mathrm{~ns}$ time bins, are due to the dark gap in the synchrotron ring filling pattern. Zooming in on the trace with a $25 \mathrm{ps}$ time bin, the $2 \mathrm{~ns}$ time structure becomes apparent, i.e., the single electron bunches in the ring, the corresponding pulsed nature of the synchrotron radiation, and, in turn, of the photoelectron signal is clearly resolved. The autocorrelation functions shown in Figure 5 are based on routinely recorded argon data for calibration purposes at a photon energy of $16 \mathrm{eV}$, at a photoelectron detection rate of $7 \times 10^{4} \mathrm{~s}^{-1}$, but loaded with electron events acting both 1 st and 2 nd events, i.e., both as start as well as stop signal in the coincidence definition for the "TOF" distribution. Thus, it is possible to carry out time resolved experiments from the ms to the ns scale.

\section{RESULTS: DISSOCIATIVE PHOTOIONIZATION OF $\mathrm{CF}_{4}$}

Among the perhalogenated methane series $\mathrm{CF}_{4}, \mathrm{CCl}_{4}$, and $\mathrm{CBr}_{4}$, only $\mathrm{CBr}_{4}{ }^{+}$can be produced by direct photoionization. ${ }^{49} \mathrm{CF}_{4}$ and $\mathrm{CCl}_{4}$ dissociatively photoionize in the Franck-Condon allowed region by halogen atom loss, ${ }^{49,50}$ although $\mathrm{CF}_{4}{ }^{+}$appears to be bound and can be produced via the dissociative photoionization of the dimers. ${ }^{51}$ The photoelectron spectroscopy and dissociative photoionization of $\mathrm{CF}_{4}$ was studied extensively, ${ }^{52,53}$ as was the kinetic energy release in the valence photoionization region. ${ }^{54-57}$ Kinugawa et al. reported anisotropic fragment ion angular distributions for $\mathrm{CCl}_{4}$ and suggested that they are indicative of dissociation on the time scale of molecular rotations. ${ }^{58}$ Such anisotropies were later confirmed by Hikosaka and Shigemasa in $\mathrm{CF}_{4}$ above a photon energy of $17 \mathrm{eV}^{59}$ The energetics of the $\mathrm{CF}_{4}+h v$ $\rightarrow \mathrm{CF}_{3}{ }^{+}+\mathrm{F}+\mathrm{e}^{-}$process is well known, ${ }^{60-62}$ and kinetic energy release analysis may yield insights into the dissociation mechanism, as was shown for the non-statistical $\mathrm{CH}_{3}$ loss in methanol cations ${ }^{25}$ or $\mathrm{F}$ loss in $\mathrm{C}_{2} \mathrm{~F}_{4}{ }^{+} .{ }^{62}$ The latest dissociative photoionization study of $\mathrm{CF}_{4}$ was reported by Garcia et al. ${ }^{10}$ who confirmed the appearance of $\mathrm{CF}_{3}{ }^{+}$at the photoionization onset, much above the calculated thermochemical dissociative photoionization threshold.

The photoelectron-photoion double velocity map imaging coincidence experiments reported here were carried out on room temperature $\mathrm{CF}_{4}$ sample in the $15.3-17 \mathrm{eV}$ photon energy range with typical count rates of $2-3 \times 10^{4} \mathrm{~s}^{-1}$ in the 16-17 eV range. Some characteristic electron and ion images as well as a TOF distribution are shown in Figure 6. The spots in the electron image are due to an only partly successful MCP cleaning procedure. Presumably, some solvent or sample traces still remained in the microchannels when high voltage was applied. However, only the central, threshold part of the electron image is used, and the kinetic energy electron background is subtracted based on a ring area around the central spot. ${ }^{28}$
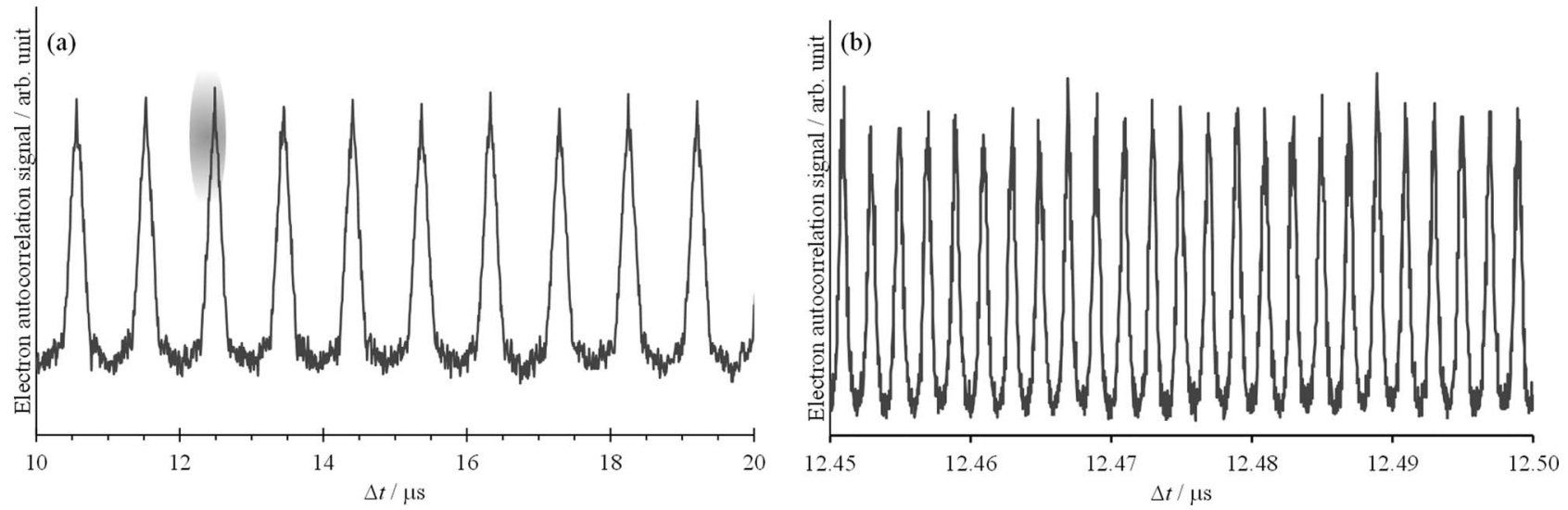

FIG. 5. (a) Argon photoelectron autocorrelation function with $8 \mathrm{~ns}$ resolution at $16 \mathrm{eV}$. The $0.96 \mu$ s periodicity is due to the dark gap in the synchrotron ring filling pattern and the $1.04 \mathrm{MHz}$ ring clock. (b) Zoom-in on the highlighted section in (a) in the 12.45-12.50 $\mu$ s range with a time resolution of $25 \mathrm{ps}$. The $2 \mathrm{~ns}$ periodicity corresponding to the electron bunches in the synchrotron ring is clearly resolved proving an adequately short flight time jitter of the photoelectrons. 

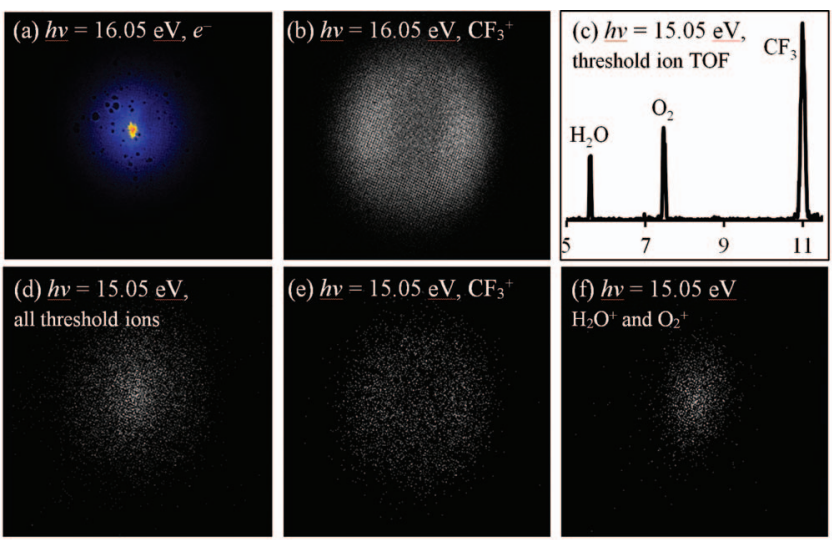

FIG. 6. (a) $\mathrm{CF}_{4}$ photoelectron image at a photon energy of $16.05 \mathrm{eV}$. The central, threshold spot on the electron image and the $\mathrm{CF}_{3}+$ TOF peak is selected to obtain the $\mathrm{CF}_{3}{ }^{+}$ion coincidence image as depicted in (b). The intensity dip in the center indicates a quite narrow kinetic energy distribution at this energy. (c) Mass spectrum shown at $15.50 \mathrm{eV}$. (d) Complete ion image corresponding to threshold electrons. (e) is obtained from (d) when only the TOF range for $\mathrm{CF}_{3}{ }^{+}$is selected. (f) Results from imaging $\mathrm{H}_{2} \mathrm{O}^{+}$and $\mathrm{O}_{2}{ }^{+}$ ions.

Linearly polarized light photoionizes the sample, and there appears to be some angular anisotropy in the high energy part of the threshold $\mathrm{CF}_{3}{ }^{+}$image at $h v=16.05 \mathrm{eV}$ in Fig. 6(b), extending the results of Hikosaka to lower photon energies and threshold photoionization..$^{59}$ The ion TOF distribution at $15.5 \mathrm{eV}$ in Fig. 6(c) shows two significant impurity peaks, $\mathrm{H}_{2} \mathrm{O}^{+}$and $\mathrm{O}_{2}{ }^{+}$, besides the dissociative photoionization product of $\mathrm{CF}_{4}: \mathrm{CF}_{3}{ }^{+}$. The complete threshold ionization ion image in Fig. 6(d) is the sum of the impurities and the sample peaks, which can be disentangled based on ion TOF discrimination. The impurity peaks in Fig. 6(f) have a thermal energy distribution, whereas the $\mathrm{CF}_{3}{ }^{+}$ion image shown in Fig. 6(e) is dominated by the kinetic energy release.

In the Franck-Condon allowed photon energy region, the neutral energy distribution is transposed onto a repulsive part of the ground electronic ion state. Nuclear forces in this energy range are such that it is tempting to assume that the dissociation takes place impulsively, i.e., on a time scale faster than intramolecular vibrational relaxation (IVR), in other words faster than the statistical redistribution of the internal energy between different degrees of freedom. ${ }^{53,63,64}$ As the ion dissociates along a symmetry axis, no rotationally excited products are expected. ${ }^{65}$ However, a constant part of the excess energy is deposited in the umbrella motion of $\mathrm{CF}_{3}{ }^{+}$, while the rest of it should be converted to kinetic energy. If the C-F bonds are supposed to be soft, $49 \%$ of the excess energy should be released. ${ }^{54}$ Thus, the kinetic energy release distribution (KERD) corresponds to the fairly narrow internal energy distribution of the neutral. As the photoionization signal appears almost $1 \mathrm{eV}$ above the dissociative photoionization threshold, the KERD could be assumed to be monoenergetic. In such cases, the single Newton sphere of fragment ions is projected onto the detector, and it can be shown that $\langle R\rangle=\pi R_{N} / 4$, where $\langle R\rangle$ is the signal weighted average radius of the image around its center and $R_{N}$ is the radius of the Newton sphere. The latter, in turn, is proportional to the momentum of the fragment ion $\mathrm{CF}_{3}{ }^{+}$, and the corresponding kinetic energy is given as $K E_{\mathrm{CF}_{3}^{+}}=\left(R_{N} / R_{\max }\right)^{2} E_{\text {max }}$, where $E_{\max }$ is the maximum range of the detector, determined to be $0.8 \mathrm{eV}$ at the extraction field of $80 \mathrm{~V} \mathrm{~cm}^{-1}$, with a corresponding Newton sphere radius of $R_{\max }=20 \mathrm{~mm}$ in our detector.

We consider the initial thermal energy in relating the kinetic energy of the fragment ion with the kinetic energy release. Because of conservation of momentum, $m\left(\mathrm{CF}_{3}^{+}\right) / m\left(\mathrm{CF}_{4}^{+}\right) \approx 69 / 88 \approx 0.784$ parts of the total initial thermal kinetic energy and $m(\mathrm{~F}) / m\left(\mathrm{CF}_{4}^{+}\right) \approx 19 / 88 \approx 0.216$ parts of the total released kinetic energy will be imparted on the $\mathrm{CF}_{3}{ }^{+}$ion. The corresponding momentum vectors will be randomly oriented in space. Let the $\mathrm{CF}_{3}{ }^{+} \mathrm{KER}$ momentum vector of magnitude $a$ be larger than the thermal momentum defined as unity, i.e., $a>1$, and be fixed in space so that it points from $(0,0,-a)$ into the origin in Cartesian coordinates. The randomly oriented thermal momentum vector then defines a unit sphere around the origin, on which the end point of the vector sum of momenta is homogeneously distributed. It can be shown that by defining $u$ as uniformly distributed in $[-1,1]$ and $\theta$ as uniformly distributed in $[0,2 \pi), x$ $=\sqrt{1-u^{2}} \cos \theta, y=\sqrt{1-u^{2}} \sin \theta$, and $z=u$ define a uniform $(x, y, z)$ distribution on the sphere. The length of the sum of momenta vector will thus be $l=\sqrt{\left(1-u^{2}\right)+(a-u)^{2}}$, and its expectation value $\langle l\rangle=\int_{-1}^{1} l(u) d u / 2=a+\frac{1}{3 a}$. Consequently, the total kinetic energy of the fragment will be

$$
K E_{\mathrm{CF}_{3}^{+}}=\left(\sqrt{K E R}+\frac{E_{\text {therm }}}{3 \sqrt{K E R}}\right)^{2}
$$

The $\mathrm{CF}_{3}{ }^{+}$kinetic energy in threshold ionization as a function of photon energy was thus determined $15.3-17.0 \mathrm{eV}$ photon energy range and is shown together with the threshold photoelectron spectrum (TPES) in Figure 7. This function can be fitted quite well below and above the first TPES peak assuming that a fraction $(f=0.43$ and $f=0.235$, respectively) of the excess energy is released in the total KERD. In neither energy regime do we find $f=0.50$ as was reported first by Powis for direct photoionization. ${ }^{54}$ Below a photon energy of $15.6 \mathrm{eV}$, the dissociation is quite impulsive, after which there is an intermediate range, and, above $16.6 \mathrm{eV}$, the kinetic energy release is almost like in a statistical dissociation. The mechanism change in the energy range of the first TPES peak is evident, and the kinetic energy release arguably corresponds to a statistical dissociation at higher energies. The lifetime of the intermediate state in the Franck-Condon allowed region of the ground ion state is, thus, long enough to allow for IVR prior to fluorine atom loss.

This also means that the assumption that the $\mathrm{CF}_{3}{ }^{+}$kinetic energy distribution is almost monoenergetic is not exact, although an acceptable approximation in the low energy regime. The derived $\mathrm{CF}_{3}{ }^{+}$kinetic energy in Figure 7 is based on the average momentum, and is underestimated for broad distributions. An accurate average kinetic energy could only be derived by distribution analysis, for which the calibration of the ion images is insufficient. However, this issue may only partly explain the discrepancy between our total KER of $0.45 \mathrm{eV}$ at $16.3 \mathrm{eV}$ and that of Creasy et al. ${ }^{57}$ and Powis ${ }^{54}$ of about $0.97 \mathrm{eV}$. A possible reason could be that neither study used 


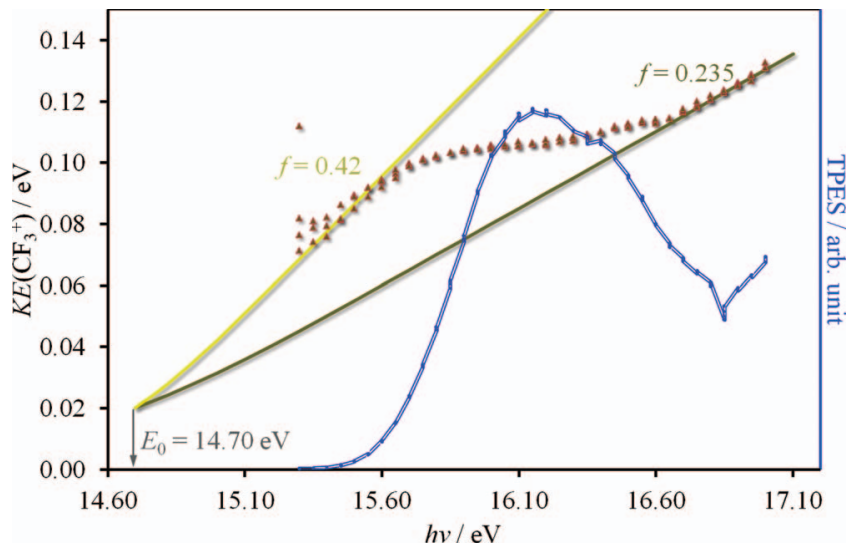

FIG. 7. Measured $\mathrm{CF}_{3}+$ fragment ion kinetic energy in threshold ionization as a function of photon energy plotted as triangles. The double line corresponds to the TPES, the single lines to kinetic energy release models. The KERD is, though not strictly speaking monoenergetic, but still characteristic of an impulsive dissociation at low photon energies, while broader at high photon energies, indicating a more statistical dissociation mechanism.

threshold ionization. In high energy direct photoionization, the photoelectron is emitted from a virtual state, and the photoion is directly obtained. In threshold photoionization, intermediate Rydberg states can always be involved. Thus, we see some indication that the threshold and direct photoionization occur via fundamentally different mechanisms.

\section{CONCLUSIONS AND OUTLOOK}

The double velocity map imaging $i^{2}$ PEPICO setup has been presented in this study together with a beamline upgrade, which includes a novel, turntable-type grazing incidence VUV monochromator with three grating positions and fast grating change and exchange. We have shown that the trigerless multiple-start/multiple-stop data acquisition technique allows for the on-the fly processing of two quadruple coincidence sets for imaging and delayed, time-of-flight coincidence analysis for mass analysis at high count rates. In addition to photoelectron photoion coincidence velocity map imaging studies, illustrated by the dissociative photoionization of $\mathrm{CF}_{4}$, we also discussed the possibility to mount grids and set up first order space focusing conditions for both positively and negatively charged species, which could allow for ion pair formation studies, as well. The flexibility with which coincidence pulse trains can be defined in our data acquisition approach was demonstrated by time-resolving processes from the ns to the ms scale. Together with our ability to measure dissociation rates in the $10^{3}-10^{7} \mathrm{~s}^{-1}$ range based on TOF peak shape analysis in the $i$ PEPICO setup, ${ }^{31}$ this allows for timeresolved studies from nanosecond to millisecond time scales, in other words over six orders of magnitude. We plan to exploit this capability in temporal analyses of reaction products in the future.

\section{ACKNOWLEDGMENTS}

We are indebted to Professor Richard P. Tuckett for inspiration and fruitful discussions. The workshop of the Paul
Scherrer Institut designed and produced the monochromator as well as the $i^{2}$ PEPICO components. We are particularly grateful to Urs Ellenberger, Markus Vitins, Herbert Kalt, Pirmin Böhler, André Schwab, Sebatian Sonderegger, Andy Keller, and Stephan Maag for their work. The beamline upgrade could not have happened without the infrastructure support and particularly the help from Rolf Wullschleger and Klemens Hoechli, as well as the vacuum and survey groups at the Swiss Light Source. The pulsed source was built by Melanie Johnson. The experimental work was carried out at the VUV beamline of the Swiss Light Source of the Paul Scherrer Institut. B.S. gratefully acknowledges the support of the Petroleum Research Fund.

${ }^{1}$ A. T. J. B. Eppink and D. H. Parker, Rev. Sci. Instrum. 68, 3477 (1997).

${ }^{2}$ D. Townsend, M. P. Minitti, and A. G. Suits, Rev. Sci. Instrum. 74, 2530 2539 (2003).

${ }^{3}$ A. J. R. Heck and D. W. Chandler, Annu. Rev. Phys. Chem. 46, 335-372 (1995).

${ }^{4}$ A. Stolow, A. E. Bragg, and D. M. Neumark, Chem. Rev. 104, 1719-1757 (2004).

${ }^{5}$ D. M. Neumark, J. Phys. Chem. A 112, 13287-13301 (2008).

${ }^{6}$ R. Dorner, V. Mergel, O. Jagutzki, L. Spielberger, J. Ullrich, R. Moshammer, and H. Schmidt-Bocking, Phys. Rep., Phys. Lett. 330, 95-192 (2000).

${ }^{7}$ M. Takahashi, J. P. Cave, and J. H. D. Eland, Rev. Sci. Instrum. 71, 13371344 (2000).

${ }^{8}$ J. Ullrich, R. Moshammer, A. Dorn, R. Dorner, L. P. H. Schmidt, and H. Schniidt-Bocking, Rep. Prog. Phys. 66, 1463-1545 (2003).

${ }^{9}$ M. Lebech, J. C. Houver, and D. Dowek, Rev. Sci. Instrum. 73, 1866-1874 (2002).

${ }^{10}$ G. A. Garcia, H. Soldi-Lose, and L. Nahon, Rev. Sci. Instrum. 80, 023102 (2009).

${ }^{11}$ P. O'Keffe, P. Bolognesi, M. Coreno, A. Moise, R. Richter, G. Cautero, L. Stebel, R. Sergo, Y. Ovcharenko, and L. Avaldi, Rev. Sci. Instrum. 82, 033109 (2011).

${ }^{12}$ X. F. Tang, X. G. Zhou, M. L. Niu, S. L. Liu, J. D. Sun, X. B. Shan, F. Y. Liu, and L. S. Sheng, Rev. Sci. Instrum. 80, 113101 (2009).

${ }^{13}$ A. Bodi, M. Johnson, T. Gerber, Z. Gengeliczki, B. Sztáray, and T. Baer, Rev. Sci. Instrum. 80, 034101 (2009).

${ }^{14}$ M. Johnson, A. Bodi, L. Schulz, and T. Gerber, Nucl. Instrum. Methods Phys. Res. A 610, 597-603 (2009).

${ }^{15}$ N. Gador, E. Samoylova, V. R. Smith, A. Stolow, D. M. Rayner, W. Radloff, I. V. Hertel, and T. Schultz, J. Phys. Chem. A 111, 11743-11749 (2007).

${ }^{16}$ P. Hockett, M. Staniforth, and K. L. Reid, Mol. Phys. 108, 1045-1054 (2010).

${ }^{17}$ K. L. Reid, Mol. Phys. 110, 131-147 (2012).

${ }^{18}$ U. J. Meierhenrich, J. J. Filippi, C. Meinert, J. H. Bredehoft, J. Takahashi, L. Nahon, N. C. Jones, and S. V. Hoffmann, Angew. Chem., Int. Ed. 49, 7799-7802 (2010)

${ }^{19}$ L. Nahon, G. A. Garcia, H. Soldi-Lose, S. Daly, and I. Powis, Phys. Rev. A 82, 032514 (2010).

${ }^{20}$ F. Gaie-Levrel, G. A. Garcia, M. Schwell, and L. Nahon, Phys. Chem. Chem. Phys. 13, 6993-7005 (2011).

${ }^{21}$ O. Plekan, M. Coreno, V. Feyer, A. Moise, R. Richter, M. de Simone, R. Sankari, and K. C. Prince, Phys. Scr. 78, 058105 (2008).

${ }^{22}$ W. A. Chupka, P. J. Miller, and E. E. Eyler, J. Chem. Phys. 88, 3032-3036 (1988).

${ }^{23}$ A. Bodi, N. S. Shuman, and T. Baer, Phys. Chem. Chem. Phys. 11, 1101311021 (2009).

${ }^{24}$ T. Baer, A. Guerrero, J. Z. Davalos, and A. Bodi, Phys. Chem. Chem. Phys. 13, 17791-17801 (2011).

${ }^{25}$ S. Borkar, B. Sztáray, and A. Bodi, Phys. Chem. Chem. Phys. 13, 1300913020 (2011)

${ }^{26}$ D. M. P. Holland, J. B. West, A. C. Parr, D. L. Ederer, R. Stockbauer, R. D. Buff, and J. L. Dehmer, J. Chem. Phys. 78, 124-130 (1983).

${ }^{27}$ S. Borkar, L. Ooka, A. Bodi, T. Gerber, and B. Sztáray, J. Phys. Chem. A 114, 9115-9123 (2010).

${ }^{28}$ B. Sztáray and T. Baer, Rev. Sci. Instrum. 74, 3763-3768 (2003).

${ }^{29}$ A. Bodi, B. Sztáray, T. Baer, M. Johnson, and T. Gerber, Rev. Sci. Instrum. 78, 084102 (2007) 
${ }^{30}$ T. Baer, B. Sztáray, J. P. Kercher, A. F. Lago, A. Bodi, C. Skull, and D. Palathinkal, Phys. Chem. Chem. Phys. 7, 1507-1513 (2005).

${ }^{31}$ B. Sztáray, A. Bodi, and T. Baer, J. Mass Spectrom. 45, 1233-1245 (2010).

${ }^{32} \mathrm{~W}$. Li, L. Poisson, D. S. Peterka, M. Ahmed, R. R. Lucchese, and A. G. Suits, Chem. Phys. Lett. 374, 334-340 (2003).

${ }^{33}$ B. Gans, G. A. Garcia, S. Boye-Peronne, J. C. Loison, S. Douin, F. GaieLevrel, and D. Gauyacq, J. Phys. Chem. A 115, 5387-5396 (2011).

${ }^{34}$ A. Mahjoub, M. Hochlaf, L. Poisson, N. Nieuwjaer, F. Lecomte, J.-P. Schermann, G. Gregoire, B. Manil, G. A. Garcia, and L. Nahon, ChemPhysChem 12, 1822-1832 (2011).

${ }^{35}$ W. R. Stevens, A. Bodi, and T. Baer, J. Phys. Chem. A 114, 11285-11291 (2010).

${ }^{36}$ W. C. Wiley and I. H. McLaren, Rev. Sci. Instrum. 26, 1150-1157 (1955).

${ }^{37}$ K. Mitsuke, H. Yoshida, and H. Hattori, Z. Phys. D: At., Mol. Clusters 27, 267-273 (1993).

${ }^{38} \mathrm{H}$. Yoshida and K. Mitsuke, J. Chem. Phys. 100, 8817-8824 (1994).

${ }^{39}$ A. Kvaran, H. Wang, and A. Logadottir, J. Chem. Phys. 112, 10811-10820 (2000).

${ }^{40}$ A. J. Yencha, D. Kaur, R. J. Donovan, A. Kvaran, A. Hopkirk, H. Lefebvrebrion, and F. Keller, J. Chem. Phys. 99, 4986-4992 (1993).

${ }^{41}$ A. Kvaran, K. Sveinbjoernsson, J. Long, and H. Wang, Chem. Phys. Lett. 516, 12-16 (2011).

${ }^{42}$ M. J. Simpson and R. P. Tuckett, Int. Rev. Phys. Chem. 30, 197-273 (2011).

${ }^{43}$ M. J. Simpson, R. P. Tuckett, K. F. Dunn, C. A. Hunniford, and C. J. Latimer, J. Chem. Phys. 130, 194302 (2009).

${ }^{44}$ S. X. Tian, Phys. Chem. Chem. Phys. 14, 6433-6443 (2012).

${ }^{45}$ P. Hemberger, M. Steinbauer, M. Schneider, I. Fischer, M. Johnson, A. Bodi, and T. Gerber, J. Phys. Chem. A 114, 4698-4703 (2010).

${ }^{46}$ M. Steinbauer, P. Hemberger, I. Fischer, M. Johnson, and A. Bodi, Chem. Phys. Lett. 500, 232-236 (2010).

${ }^{47}$ M. Steinbauer, P. Hemberger, I. Fischer, and A. Bodi, ChemPhysChem 12, 1795-1797 (2011).

${ }^{48}$ See supplementary material at http://dx.doi.org/10.1063/1.4742769 for manuscript reference and examples.
${ }^{49}$ A. S. Werner, B. P. Tsai, and T. Baer, J. Chem. Phys. 60, 3650-3657 (1974).

${ }^{50}$ T. A. Walter, C. Lifshitz, W. A. Chupka, and J. Berkowitz, J. Chem. Phys. 51, 3531-3536 (1969).

${ }^{51}$ G. Hagenow, W. Denzer, B. Brutschy, and H. Baumgartel, J. Phys. Chem. 92, 6487-6488 (1988)

${ }^{52}$ D. M. P. Holland, A. W. Potts, A. B. Trofimov, J. Breidbach, J. Schirmer, R. Feifel, T. Richter, K. Godehusen, M. Martins, A. Tutay, M. Yalcinkaya, M. Al-Hada, S. Eriksson, and L. Karlsson, Chem. Phys. 308, 43-57 (2005).

${ }^{53}$ D. M. Smith, R. P. Tuckett, K. R. Yoxall, K. Codling, P. A. Hatherly, J. F. M. Aarts, and M. Stankiewicz, J. Chem. Phys. 101, 10559-10575 (1994).

${ }^{54}$ I. Powis, Mol. Phys. 39, 311-327 (1980).

${ }^{55}$ B. Brehm, R. Frey, A. Küstler, and J. H. D. Eland, Int. J. Mass Spectrom. Ion Phys. 13, 251-260 (1974).

${ }^{56}$ I. G. Simm, C. J. Danby, J. H. D. Eland, and P. I. Mansell, J. Chem. Soc., Faraday Trans. 2 72, 426-434 (1976).

${ }^{57}$ J. C. Creasey, H. M. Jones, D. M. Smith, R. P. Tuckett, P. A. Hatherly, K. Codling, and I. Powis, Chem. Phys. 174, 441-452 (1993).

${ }^{58}$ T. Kinugawa, Y. Hikosaka, A. M. Hodgekins, and J. H. D. Eland, J. Mass Spectrom. 37, 854-857 (2002).

${ }^{59}$ Y. Hikosaka and E. Shigemasa, J. Electron Spectrosc. Relat. Phenom. 152, 29-32 (2006).

${ }^{60}$ A. Bodi, A. Kvaran, and B. Sztáray, J. Phys. Chem. A 115, 13443-13451 (2011).

${ }^{61}$ J. Csontos, Z. Rolik, S. Das, and M. Kallay, J. Phys. Chem. A 114, 1309313103 (2010).

${ }^{62}$ J. Harvey, A. Bodi, R. P. Tuckett, and B. Sztáray, Phys. Chem. Chem. Phys. 14, 3935-3948 (2012).

${ }^{63}$ G. K. Jarvis, K. J. Boyle, C. A. Mayhew, and R. P. Tuckett, J. Phys. Chem. A 102, 3230-3237 (1998).

${ }^{64}$ G. K. Jarvis, K. J. Boyle, C. A. Mayhew, and R. P. Tuckett, J. Phys. Chem. A 102, 3219-3229 (1998).

${ }^{65}$ T. Baer and W. L. Hase, Unimolecular Reaction Dynamics: Theory and Experiments (Oxford University Press, New York, 1996). 